\title{
The Huygens Mission to Titan: Overview and status
}

\author{
J.-P. Lebreton
}

Research and Scientific Support Department of ESA, ESTEC, P.O. Box 299, 2200 AG Noordwijk, The Netherlands

D. L. Matson

Jet Propulsion Laboratory, California Institute of technology, 4800 Oak Grove Drive, Pasadena, CA 91109, U.S.A.

Huygens is an entry probe designed to descend under parachute through the atmosphere of Titan, Saturn's largest moon. The Huygens Probe is provided by the European Space Agency (ESA) for the Cassini/Huygens mission to Saturn and Titan. The Huygens mission will be conducted on the 3rd Orbit around Saturn. The probe will be released around December 25, 2004 for entry in Titan on January 14,2005. This paper provided an overview of the Huygens mission. The status of the probe and of the mission was reviewed, and opportunities for Titan observations by the Orbiter during the first two orbits were discussed. The Cassini/Huygens mission is a joint undertaking by NASA and ESA, with ASI as a partner via a bilateral agreement with NASA. 